\title{
Changes in Qualities and Quantities of Consecutively Ejaculated Feline Semen
}

\author{
Hiromichi OBA $^{1) *}$, Youta SAITO ${ }^{1)}$, Tatsuji MIZUTANI ${ }^{1)}$, Mari TOYONAGA ${ }^{1)}$ and Toshihiko TSUTSUI ${ }^{1)}$

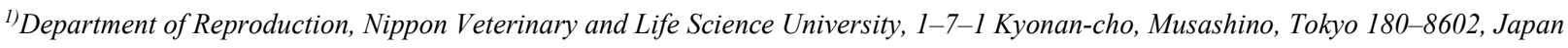

(Received 21 July 2010/Accepted 22 September 2010/Published online in J-STAGE 6 October 2010)

ABSTRACT. Cats show repeated copulation, but changes in semen qualities and quantities with repetition of ejaculation have not previously been clarified. We collected semen 4 times consecutively from 5 cats using the artificial vagina method and observed the semen qualities and quantities. No significant changes were noted in the semen volume, frequency of abnormal sperm or incidence of immature sperm, but the number of sperm and sperm motility and viability decreased with repetition, and in particular, the number of sperm in the first semen accounted for $55.0 \%$ of the total number in the 4 consecutive ejaculations, showing a significant difference from those in the 2 nd4th semen $(P<0.01)$

KEY WORDS: consecutively ejaculation, feline, semen.

J. Vet. Med. Sci. 73(2): 245-247, 2010

Cats are copulatory ovulators that show repeated copulation, but the ovulation induction rate per single copulation has been reported to be low by Concannon et al. [1], Wildt et al. [11], Glover et al. [4] and us [10]. Concannon et al. [1] observed the relationship between the number of copulations and plasma LH level after copulatory stimulation. Plasma LH elevation after a single copulation was insufficient to induce ovulation in some cats, and $50 \%(9 / 18)$ of cats in their experiment did not ovulate. However, the plasma LH level rose after copulation 4 times or more, and the ovulation induction rate reached $100 \%(36 / 36)$. Regarding the relationship between the number of copulations and conception, Glover et al. [4] reported that only one (25.0\%) of 4 cats in which ovulation was induced by a single copulation was fertilized, but the conception rate was $71.4 \%(5 / 7)$ in cats that copulated 2-3 times. We also performed a similar experiment [10]; in that experiment, the conception rate was $37.5 \%(6 / 16)$ in cats in which ovulation was induced by a single copulation, but it increased to $94.1 \%$ (16/17) after 3 copulations, as reported by Glover et al. [4]. An insufficient number of sperm per copulation was assumed to be the reason for the low conception rate after a single copulation.

However, changes in the qualities and quantities of consecutively ejaculated feline semen have not been clarified.

Thus, we collected feline semen from 4 consecutive ejaculations using the artificial vagina method (AVM) and investigated changes in the semen qualities and quantities.

Male cats bred and maintained in our colony were used in this experiment. Five 3.2-4.6-year-old cats weighing 4.2$5.3 \mathrm{~kg}$ were used. These cats showed normal copulatory capability and fertility. The animals were maintained in a room in which the temperature was adjusted to $23 \pm 2{ }^{\circ} \mathrm{C}$ and were kept individually in cages measuring $60 \times 90 \times 120$ $\mathrm{cm}$. The animal room was kept under a 12-hr lighting cycle. The animals were given commercial dry food (Hill's Feline

\footnotetext{
* Correspondence to: OBa, H., Department of Reproduction, Nippon Veterinary and Life Science University, 1-7-1 Kyonan-cho, Musashino, Tokyo 180-8602, Japan. e-mail: oba1111@athena.ocn.ne.jp
}

Maintenance, Hill's Pets Nutrition, Inc., Topeka, KS, U.S.A.) and water ad libitum. The female estrous cats maintained in our colony were appropriately used for semen collection.

This study was conducted in conformity with the animal study guidelines of Nippon Veterinary and Life Science University.

Semen was collected using an artificial vagina (AV) for cats that has previously been reported [8].

The AV was exchanged for a new one, and collection was repeated after about $10 \mathrm{~min}$. Cats ejaculated 4 times consecutively. This repeated ejaculation experiment was performed 4 times at 2-week resting intervals.

The semen volume was measured using a sample tube with $50 \mu l$ graduations. Sperm motility was represented by ratio (\%) of sperm actively moving forward using a test plate for semen property examination (FA225, Fujihira Industry, Co., Ltd., Tokyo, Japan). The number of sperm, sperm viability, sperm abnormality and frequency of immature sperm were examined as previously reported [5].

The significance of semen quality and quantity was analyzed among the groups by employing two-way ANNOVA and the Tukey-Kramer test. A $P$-value of less than 0.05 was regarded as significant.

Changes in the qualities of consecutively ejaculated semen are presented as the means \pm SE of the parameters in Fig. 1. The consecutive ejaculation experiment was performed 4 times using 5 male cats. No reduction of sexual or mounting activity levels was noted in any male cat throughout the study period.

The volume of semen in each consecutive ejaculation only slightly decreased. The volume of the first ejaculated semen was $42.8 \pm 5.6 \mu l$ (mean $\pm \mathrm{SE})$, and the volume of the fourth semen was $39.8 \pm 2.5 \mu \mathrm{l}$. The mean number in the first ejaculated semen was $8.3 \pm 0.4 \times 10^{7}$, but it significantly decreased to $2.9 \pm 0.4 \times 10^{7}$ in the second semen, about $35 \%$ of that in the first semen $(P<0.01)$. The number of sperm in the third and fourth semen was $2.1 \pm 0.1 \times 10^{7}$ and $1.5 \pm 0.1 \times 10^{7}$, respectively, significantly smaller than 

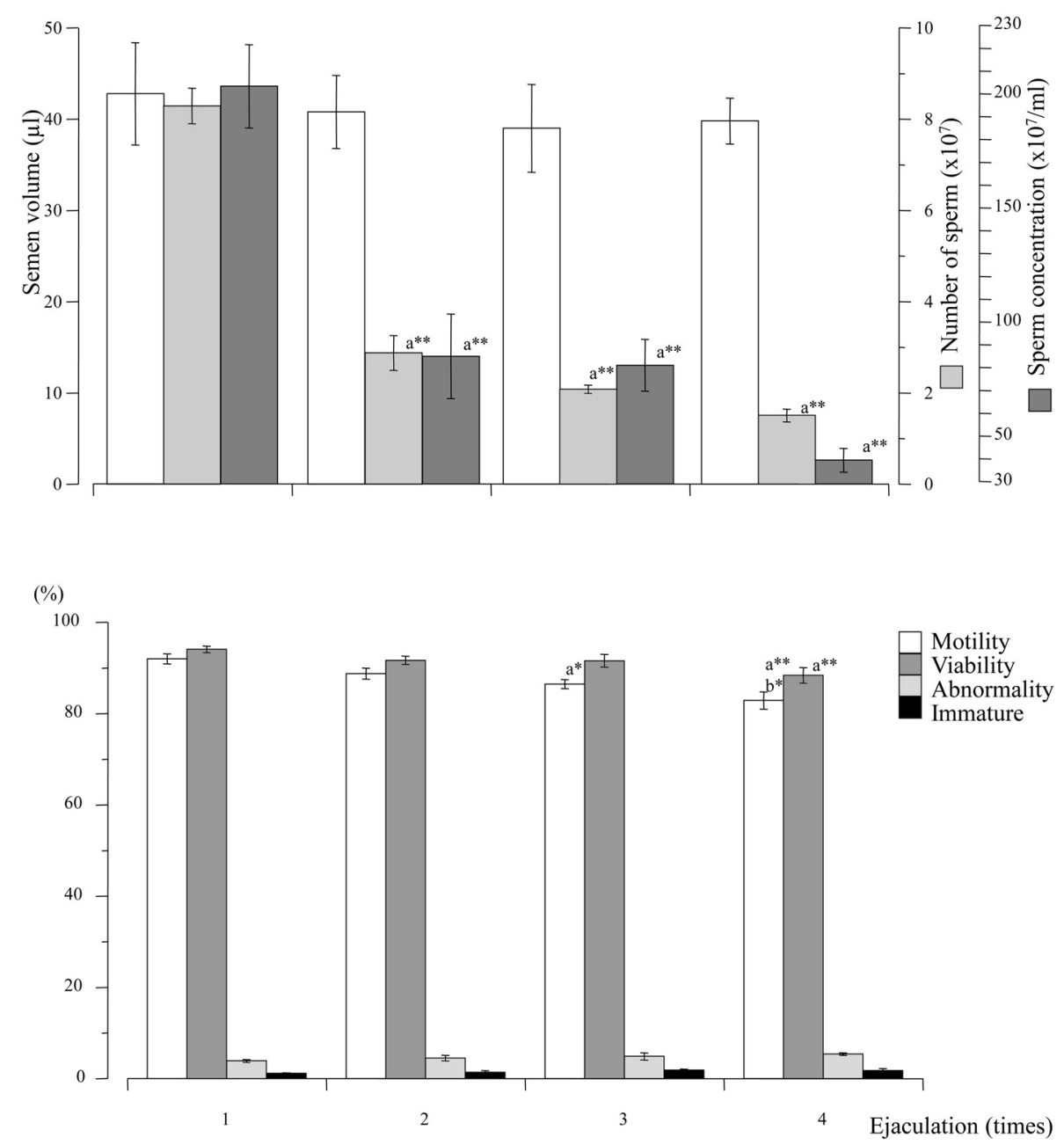

Fig. 1. Changes in the qualities of consecutively ejaculated semen $(m \pm S E)$. a: Differed significantly from the first ejaculated semen. b: Differed significantly from the first ejaculated semen $(*: P<0.05$, $* *: P<0.01)$

the number in the first semen $(P<0.01)$. Furthermore, there was a significant difference between the second and fourth semen $(P<0.05)$. The total number of sperm in the 4 consecutively ejaculated semen samples was $14.3 \pm 2.0 \times 10^{7}-15.2$ $\pm 2.6 \times 10^{7}$ in the individual cats, and the mean was $14.8 \pm$ $0.3 \times 10^{7}$. The mean percentages of the total number of sperm for the 1 st, 2 nd and 3 rd semen were $55.0 \pm 3.1,20.8$ \pm 2.5 and $14.3 \pm 0.4 \%$, respectively, showing that the number in the first 2 samples accounted for $75.8 \%$ of the total number.

The mean sperm motilities were $92.0 \pm 1.1$ and $88.8 \pm$ $1.2 \%$ in the 1 st and 2 nd semen, respectively, showing no significant difference, but those in the 3rd and 4th semen were $86.5 \pm 1.0$ and $82.9 \pm 1.9 \%$, respectively, showing significant reductions from that in the 1 st semen $(P<0.01$, respectively). There was also a significant difference between the third and fourth semen $(P<0.05)$. The sperm viability slightly decreased as ejaculation was repeated, but the differences were not significant until the $3 \mathrm{rd}$ ejaculation. In the 4 th semen, the mean viability was $88.4 \pm 1.7 \%$, showing a significant reduction from that in the 1 st semen $(94.1 \pm$ $1.1 \% ; P<0.05)$. The frequency of abnormal sperm and incidence of immature sperm slightly increased with the repetition of ejaculation, but the differences were not significant.

Since semen collection using the AVM requires the training of male cats and presence of estrous female cats at the time of sampling, changes in the qualities and quantities of repeatedly ejaculated semen have not previously been reported.

Regarding the number of sperm required to achieve conception in cats, only our findings on intravaginal insemination (IVI) using fresh semen are available, in which the conception rates achieved through insemination with 2,4 and $8 \times 10^{7}$ sperm were $6 \%(1 / 16), 33.3 \%(6 / 18)$ and $77.8 \%$ (7/9), respectively, suggesting that the number of sperm required to achieve conception by IVI is $8 \times 10^{7}$ [9]. The 
number of sperm in the 1 st semen varied from $4.6 \times 10^{7}$ to $10.5 \times 10^{7}$ among the animals, and the mean was $8.3 \pm 0.4 \times$ $10^{7}$. The mean was similar to the number required to achieve conception by IVI, but the number was smaller than this in 2 of the 5 cats $\left(6.5 \times 10^{7}\right.$ and $\left.4.6 \times 10^{7}\right)$.

The volume and number of sperm in feline semen from the 1st ejaculation collected employing the AVM have been reported to be $40 \mu l$ and $5.7 \times 10^{7}, 33.8 \pm 4.8$ (SE) $\mu l$ and 6.1 $\pm 1.3 \times 10^{7}, 60 \mu l$ and $6.1 \pm 1.6 \times 10^{7}, 20 \mu l$ and $3.0 \pm 1.2 \times$ $10^{7}$ and $51 \mu l$ and $4.3 \pm 0.3 \times 10^{7}$ in studies performed by Sojka et al. [7], Platz et al. [6], Dooley \& Pineda [2], Dooley et al. [3] and us [8], respectively. The mean semen volume was $20-60 \mu l$ in these 5 reports, showing marked variation. The number of sperm also varied from $3.0 \times 10^{7}$ to $6.1 \times 10^{7}$. In the present study, the mean semen volume was $43.4 \pm 5.1$ $\mu l$, which was similar to that in previous reports, but the mean number of sperm was greater $\left(8.3 \pm 0.4 \times 10^{7}\right)$. The improvement of spermatogenesis brought about by repeated semen collection may have been the reason for the higher number of sperm in our cats.

The results of the present study suggest that the total number of sperm contained in semen ejaculated several times is necessary to achieve a high conception rate by coitus in cats.

\section{REFERENCES}

1. Concannon, P. W., Hodgson, B. and Lein, D. 1980. Reflex LH in release ini estrous cats following single and multiple copulations. Biol. Reprod. 23: 111-117.

2. Dooley, M. P. and Pineda, M. H. 1986. Effect of method of collection of seminal characteristics of the domestic cat. Am. J.
Vet. Res. 47: 286-292.

3. Dooley, M. P., Pineda, M. H., Hopper, J. G. and Hsu, W. H. 1991. Retrograde flow of spermatozoa into the urinary bladder of cats during electoroejaculation, collection of semen with an artificial vagina, and mating. Am. J. Vet. Res. 52: 687-691.

4. Glover, T. E., Watson, P. E. and Bonney, R. C. 1985. Observation on variability in LH release and fertility during oestrus in the domestic cat (Felis Catus). J. Reprod. Fertil. 23: 111-117.

5. Kawakami, E., Tsutsui, T., Yamada, Y. and Yamauchi, M. 1984. Cryptorchism in the dog; eccurence of cryptorchidism and semen quality in the cryptorchid dog. Jpn. J. Vet. Sci. 46: 303-308.

6. Platz, C. C., Wildt, D. E. and Seager, S. W. J. 1978. Pregnancy in the domestic cat after artificial insemination with previously frozen spermatozoa. J. Reprod. Fertil. 52: 279-282.

7. Sojka, N. J., Jennings, L. L. and Hamner, C. E. 1970. Artificial insemination in the cat (felis catus L.) Lab. Anim. Care 20: 198-204.

8. Tanaka, A., Kuwabara, S., Takagi, Y., Nakagawa, K., Fujimoto, Y., Murai, M. and Tsutsui, T. 2000. Effect of ejaculation intervals on semen quality in cats. J. Vet. Med. Sci. 62: 11571161.

9. Tanaka, A., Takagi, Y., Nakagawa, K., Fujimoto, Y., Hori, T. and Tsutsui, T. 2000. Artificial intravaginal insemination using fresh semen in cats. J. Vet. Med. Sci. 62: 1163-1167.

10. Tsutsui, T., Higuchi, C., Soeta, M., Oba, H., Mizutani, T. and Hori, T. 2009. Plasma LH, ovulation and conception rates in cats mated once or three times on different days of oestrus. Reprod. Dom. Anim. 44: 76-78.

11. Wildt, D. E., Seager, S. W. J. and Chakraborty, P. K. 1980. Effect of copulatory stimuli on incidence of ovulation and on semen lutenizing hormone in the cat. Endocrinology 107: 1212-1217. 